\title{
OPTICAL-RADIO REFERENCE TIES
}

\author{
J. KOVALEVSKY \\ Observatoire de la Côte d'Azur, CERGA \\ Grasse, France
}

In the course of the Hipparcos data reduction, the system of coordinates obtained is the result of complicated and partly random processes. It is therefore an arbitrary system which could not be retained. It was decided to compute a rotation $\epsilon$ at epoch (1991.25) and a time-dependent rotation $\omega$ (or spin) such that, when applied, it would be a realisation of the IAU extragalactic reference system as defined in 1991 and implemented by IERS in 1995.

A working group was set up by the Hipparcos Science Team with the task to provide these rotations from all possible techniques. Actually eleven independent teams provided a solution. One can group them in four categories.

1. Radio techniques included VLBI observations with the US Deep Space Network, VLBA and the European VLBI Network, and connected radio interferometers MERLIN and VLA. While VLBI provided a solution for both $\epsilon$ and $\omega$, the other techniques allowed to get only $\epsilon$.

2. Medium and small field astrometry including the optical positions of compact sources (Hamburg and USNO), and observations with the HST which both provided solutions for $\epsilon$ only and comparisons of plates taken at large intervals of time (Bonn and Potsdam) which gave solutions for $\omega$ only.

3. Star catalogues refered to galaxies: Lick Northern proper motion program, Yale Southern proper motion program, and KSZ catalogue. Yale University, ARI in Heidelberg and Kiev Observatory yielded solutions for $\omega$.

4. Comparison of Earth rotation parameters as obtained by VLBI and by a reduction of ground-based determinations with Hipparcos data (Prague Observatory) gave components of $\epsilon$ and $\omega$ in the equator.

I. M. Wytrzyszczak, J. H. Lieske and R. A. Feldman (eds.),

Dynamics and Astrometry of Natural and Artificial Celestial Bodies, 447, 1997.

(C) 1997 Kluwer Academic Publishers. Printed in the Netherlands. 
The relative weighting and the final determination of $\epsilon$ and $\omega$ were performed by two different methods in CERGA and Lund Observatory. The adopted uncertainties achieved were \pm 0.6 mas for $\epsilon$ and \pm 0.25 mas for $\omega$.

The work presented here will be described in detail in Astronomy and Astrophysics journal under the title "The Hipparcos Catalogue as a realisation of the extragalactic reference system" with the following co-authors:

J. Kovalevsky, L. Lindegren, M.A.C. Perryman, P.D. Hemenway, K. J. Johnston, V.S. Kislyuk, J.F. Lestrade, L.V. Morrison, I. Platais, S. Röser, E. Schilbach, H.-J. Tucholke, C. de Vegt, J. Vondrák, F. Arias, A.M. Gontier, F. Arenou, P. Brosche, D. R. Florkowski, S. T. Garrington, S. Hirte, V. Kozhurina-Platais, R. A. Preston, C. Ron, S.P. Rybka, N. Zacharias. 